\title{
Persistence of chicken anemia virus antigen and inclusions in spontaneous cases of Marek's disease visceral lymphomas in broiler chickens at slaughterhouses
}

\author{
Mohamed Sabry AHMED ${ }^{1-3)}$, Hiroki ONO ${ }^{2)}$, Jun SASAKI ${ }^{2)}$, Kenji OCHIAI ${ }^{1,2)}$ and Masanobu GORYO ${ }^{1,2) *}$ \\ 1) Department of Pathogenic Veterinary Science, The United Graduate School of Veterinary Sciences, Gifu University, 1-1 Yanagido, \\ Gifu 501-1193, Japan \\ ${ }^{2)}$ Department of Veterinary Pathology, Faculty of Agriculture, Iwate University, 3-18-8 Ueda, Iwate 020-8550, Japan \\ 3) Department of Poultry Diseases, Faculty of Veterinary Medicine, South Valley University, Qena 83523, Egypt
}

(Received 29 October 2015/Accepted 25 January 2016/Published online in J-STAGE 18 February 2016)

ABSTRACT. The chicken anemia virus (CAV) and Marek's disease virus (MDV) infect chickens worldwide; a single or dual infection by these viruses has a great impact on poultry production. In the present study, we examined the existence of CAV antigen and its inclusions in Marek's disease (MD) lymphomas in chickens in the slaughterhouses of Iwate prefecture, Japan. Forty-nine spleens and 13 livers with different degrees of nodular lesions were histopathologically examined at our laboratory. Grossly, the tested organs showed various sizes and anatomical architectures. Based on the cellular morphology and the infiltrative nature of the neoplastic lymphocytes, MD was confirmed in $76 \%(37 / 49)$ of the spleens and $92 \%(12 / 13)$ of the livers. The lesions of MD, according to the pattern of lymphocytic accumulation in the affected organs, were divided into multifocal, coalesced and diffuse. CAV intranuclear inclusion bodies were detected within the small and the large bizarre lymphocytes of the MD lymphomas in 2 livers and 9 spleens, and the immunostaining test for CAV confirmed the persistence of CAV antigens and inclusions in the neoplastic cells. This study demonstrated the persistence of CAV infection within the neoplastic cells of naturally occurring MD lymphomas in chickens.

KEY WORDS: chicken anemia virus, co-infection, inclusion body, lymphoma, marek's disease

doi: 10.1292/jvms.15-0615; J. Vet. Med. Sci. 78(5): 825-829 2016

Marek's disease (MD) is a worldwide lymphoproliferative disease affecting chickens and is caused by the Marek's disease virus (MDV). It is considered an economically important virus-induced neoplastic disease affecting poultry $[8,19]$. The disease is typically characterized by transformed T-cell lymphomas in the skin, nerves and visceral organs [3]. MD infected chickens show a wide variety of clinical forms; the acute form is one of the most virulent forms, which may affect 6-week-old chickens. The loss of chickens due to the acute form of MD commonly occurs between 3-6 months of age [18]. Young unvaccinated layer chickens at 3-4 weeks of age or prior to reaching the age of egg production [13] and young or adult commercially vaccinated chickens are also susceptible to this form [23]. MDV infection is often subclinical and associated with lymphomas in various organs [18]. The lymphomatous lesion of MD is one of the most important causes of carcass condemnation in slaughterhouses [18]. MD visceral lymphomas either develop as multiple masses or diffuse enlargement of the affected organs; sometimes the size of the organs can be several times its normal size [19].

Chicken anemia virus (CAV) is the ubiquitous cause of immunosuppressive disease in chickens [20]. Although

\footnotetext{
*Correspondence to: Goryo, M., Current Address: Department of Veterinary Pathology, Faculty of Agriculture, Iwate University, 3-18-8 Ueda, Iwate 020-8550, Japan. e-mail: goryo@iwate-u.ac.jp (C)2016 The Japanese Society of Veterinary Science

This is an open-access article distributed under the terms of the Creative Commons Attribution Non-Commercial No Derivatives (by-nc-nd) License $<$ http://creativecommons.org/licenses/by-nc-nd/4.0/>.
}

CAV has been recognized since 1979 [24] as a pathogen that mainly causes disease in chickens before 3 weeks of age, its true impact on the poultry industry has not yet been fully evaluated [17]. CAV infection in chicks in the first 3 weeks of age is characterized by severe anemia, bone marrow hypoplasia, severe immunosuppression and high mortality due to secondary bacterial or viral infections [20]. CAV infection in older chickens at or after 3 weeks of age is often subclinical due to the formation of CAV neutralizing antibodies, which prevent the appearance of clinical symptoms $[9,15$, 21]; however, these antibodies have no effect against the infection or the transmission of infection and against the immunosuppressive effect of the virus [22]. In cases of coinfection, the subclinical form of CAV infection may influence the severity of the other infection $[6,10,11]$.

Both MDV and CAV infections cause great economic losses in poultry production by causing either single or dual infections. In addition, when co-infection of these 2 viruses occurs with other pathogens, they have either a direct effect on poultry production as a result of tumor formation, anemia and delayed growth, or an indirect effect that aggravates other diseases by immunosuppression [7]. The experimental co-infection of MDV and CAV in 1-day-old [16] or 4-weekold chicks [10] is well documented; however, there is a lack of reports regarding naturally occurring co-infections of $\mathrm{CAV}$ and MDV at older ages, or regarding the mechanism of infection and the relationship between these viruses. Therefore, the aim of our study was to examine the existence of CAV antigens and its inclusions within naturally occurring MD lymphomas in chickens at slaughterhouses. 


\section{MATERIALS AND METHODS}

Samples: A total of 62 formalin fixed organs (49 spleens and 13 livers) were collected from the slaughterhouses in Iwate prefecture, Japan. These were examined at our laboratory. Most chickens were around 60 days old; however, the age of some chickens was unidentified.

Gross examination: The diameters and weight of the organs were recorded. The severity of the gross lesions, based on the size and distribution of the lymphomatous lesions in the affected organs, was scored as follows: $(-)$ score for the absence of lymphomatous lesions and no changes in the anatomical architecture of the organs; $(+)$ for solitary distributed mild lymphomatous lesions of sizes in the range of 2-3 $\mathrm{mm}$ and without changes in the anatomical architecture of the organs; $(2+)$ for solitary distributed moderate lymphomatous lesions with sizes in the range of 3-5 $\mathrm{mm}$ and with slight changes in the anatomical architecture of the affected organs; $(3+)$ for diffusely distributed severe lymphomatous lesions of $>5 \mathrm{~mm}$ in size and with noticeable changes in the anatomical architecture of the affected organs.

Histopathological examination: For histopathological examination, the organs were cut into slices and fixed with $10 \%$ neutral buffered formalin for 2 days, following which they were dehydrated and embedded in paraffin wax. All the sections were cut approximately $4 \mu \mathrm{m}$ thick and stained with hematoxylin and eosin.

Immunohistochemistry: Rabbit anti-RAV2 antibody was provided by Dr. Tsukamoto (National institute of Animal Health, Tsukuba, Japan) and was used to detect avian leukosis virus (ALV) infected neoplastic cells by detecting the ALVgs-antigen. CAV antiserum was used to detect CAV antigen; it was prepared by intramuscular inoculation of $0.1 \mathrm{~m} l$ of MDCC-MSB1 culture supernatant containing $10^{7.5}$ mean tissue culture infective dose of the MSB1-TK5803 strain into 3 -week-old specific pathogen free chicks, followed by a second inoculation 2 weeks later. The sera were then collected 4 weeks after the second inoculation inactivated in a water bath for $30 \mathrm{~min}$ at $56^{\circ} \mathrm{C}$ and stored at $-80^{\circ} \mathrm{C}$.

Four $\mu \mathrm{m}$ thick paraffin sections were deparaffinized in xylene and rehydrated in graded alcohol. Antigen retrieval was performed by autoclaving the sections in a $10 \mathrm{mM}$ citrate buffer solution, $\mathrm{pH}$ 6. The endogenous peroxidase was blocked by treating the sections with $0.3 \% \mathrm{H}_{2} \mathrm{O}_{2}$ solution in methanol for $30 \mathrm{~min}$ at room temperature. Subsequently, the sections were treated with diluted normal goat serum (Vector Lab., Peterborough, U. K.) in phosphate-buffered saline $(1: 10)$ for $30 \mathrm{~min}$ to block nonspecific reactions.

The sections used to detect the ALVgs-antigen were covered by the primary antibody, Rabbit anti-RAV2 $(1: 10,000)$, while the sections used to detect CAV were covered with CAV antisera (1:100). The sections were kept overnight in a humid chamber at $4^{\circ} \mathrm{C}$. After washing with Tris-buffered saline, the sections were covered with a secondary antibody. The sections used to detect CAV were covered with goat polyclonal anti-chicken IgG conjugated to horseradish peroxidase (HRP) enzyme (BETHYL lab. Inc., Montgomery, AL, U.S.A., diluted 1:200). The labeled streptavidin-biotin- peroxidase complex technique using the LSAB System-HRP kit (Dako North America, Inc., Carpinteria, CA, U.S.A.) was used to detect the ALVgs-antigen.

The peroxidase was activated by using 3, $3^{\prime}$-diaminobenzidine (Dako North America, Inc.). Finally, the sections were counterstained with hematoxylin for $30 \mathrm{sec}$.

\section{RESULTS}

Gross findings: The tested organs were of various weights, shapes and sizes; the weight of the spleens ranged from 2.9-150 g, and the weights of the livers ranged from 58-214 g. There were white nodules of variable sizes in $49 \%(24 / 49)$ of the tested spleens and $39 \%(5 / 13)$ of the tested livers (Fig. 1A and 1B).

On the cut surface, 61\% (30/49) of the tested spleens showed a clear marbling appearance due to the presence of white colored nodular masses of different sizes in the splenic parenchyma (Fig. 1A). Some of these nodules were coalescent and difficult to distinguish from the splenic parenchyma, while other nodules showed a clear demarcation from the splenic parenchyma in the form of a zone of hemorrhagic and necrotic areas. The findings on the cut surface of the liver were the same as that on the cut surface of the spleen; however, the nodules in the liver were totally demarcated from the hepatic parenchyma, and the hepatic surface was uneven due to the presence of these nodules (Fig. 1B). Conversely, some organs appeared to be normal on the cut surface.

The severity of the gross lesions on the surface and cut surface of the tested organs scored as follows: 17 organs, which included 11 spleens and 6 livers, scored 3+; 16 organs, which included 12 spleens and 4 livers, scored 2+; 9 organs, which included 6 spleens and 3 livers, scored +; and the remaining 20 spleens with no significant gross lesions scored as -.

Histopathological findings: The affected spleens showed neoplastic lymphocytic aggregations of various sizes ranging from focal lesions to extensive lymphoma, and these aggregations often included the periarterial lymphoid sheath and the perivenular lymphoid tissue, and appeared as a compressed red pulp. The normal splenic architecture was replaced by neoplastic cells. These aggregations were observed in $76 \%$ $(37 / 49)$ of the spleens and $92 \%(12 / 13)$ of the livers. The neoplastic lymphocytes severely infiltrated the hepatic parenchyma with severe dilatation of the hepatic sinusoids. Each lesion was composed of small mature lymphocytes, large bizarre lymphocytes, lymphoblasts, tangible body macrophages and pyknotic lymphocytes. The mitotic count ranged from 3/ high power field (HPF) in mild lesions to 10/HPF in severe lesions. The large bizarre lymphocytes, which were 2-3 times larger than the other lymphoid cells with a large nucleus and moderate cytoplasm, were located mainly in the center of the neoplastic lymphoid aggregations; however, sometimes, these cells were observed at the periphery of the lymphoid mass. These lymphocytic aggregations were confirmed as MD lymphomas. On the other hand, multiple and small eosinophilic intranuclear inclusion bodies were observed, oc- 

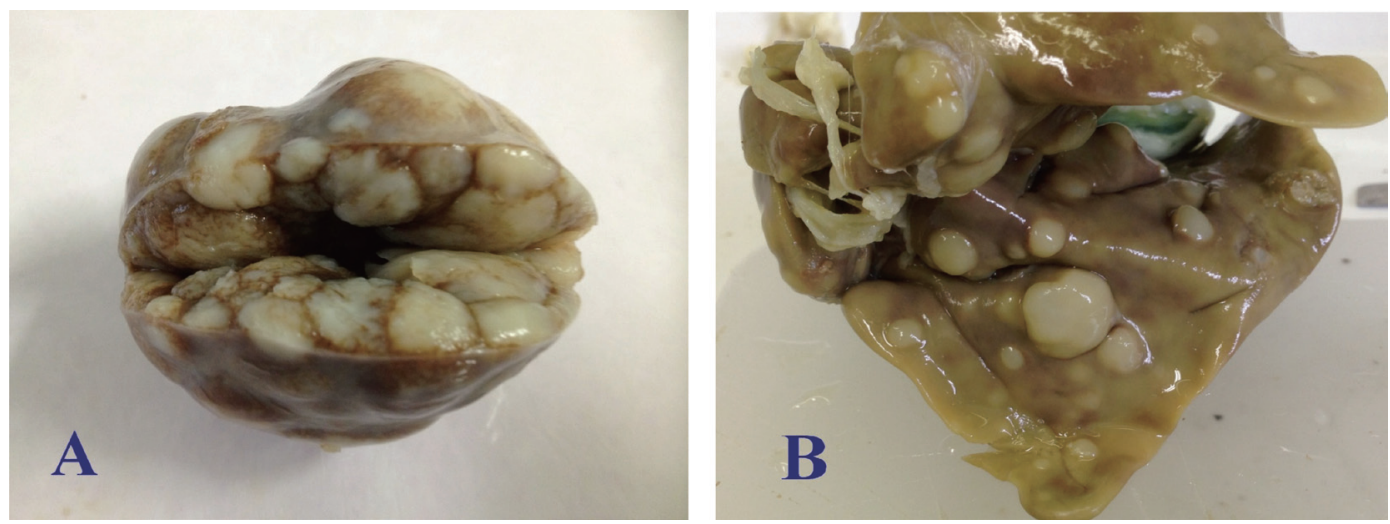

Fig. 1. The gross pathology of the spleen (A) and liver (B) of a 60-day-old chicken shows alteration in the anatomical architecture due to the presence of variable sized nodules. On cut surface, the spleen (A) shows a clear marbling appearance due to the presence of white to grayish creamy nodular masses which are embedded within the parenchyma. The liver (B) surface appears uneven due to the presence of raised white nodular mass.
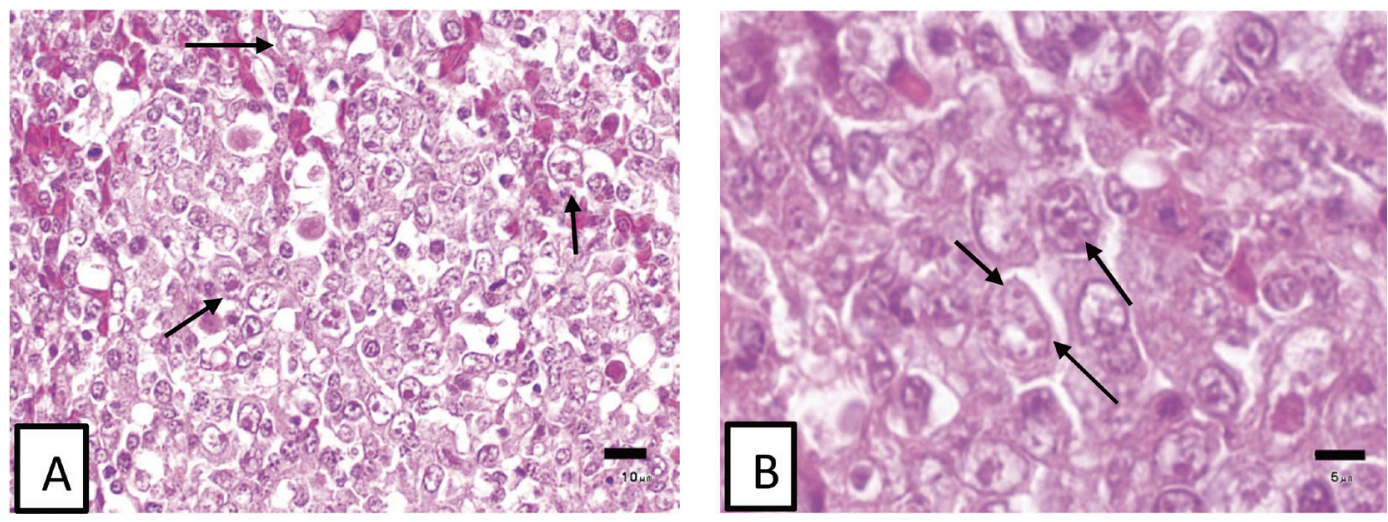

Fig. 2. (A) The spleen shows variable sized tumorous proliferations of large bizarre shaped lymphoid cells with large nuclei and moderate cytoplasm (arrows) located at the center or periphery of the lymphoid mass; bar $=10 \mu \mathrm{m}$. (B) The large bizarre shaped lymphocytes of the MD lymphoma in the spleen show small, multiple and variable sized eosinophilic inclusions in their nuclei (arrows). Bar $=5 \mu \mathrm{m}$; hematoxylin and eosin staining.
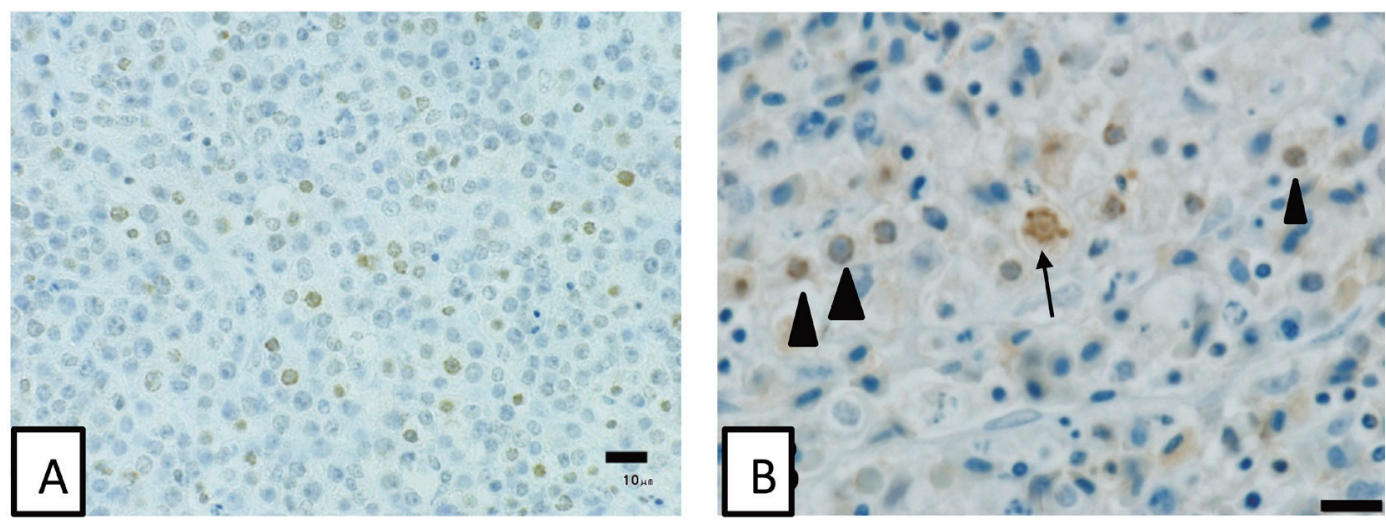

Fig. 3. The immunohistochemistry of the spleen using CAV antiserum. (A) CAV antigen was detected within the MD lymphoma in the large bizarre and small lymphocytes. Bar $=10 \mu \mathrm{m}$. (B) CAV antigen was detected as intranuclear fine granular inclusions within the large bizarre shaped lymphocytes (arrow) and the small lymphocytes (arrowheads). $\mathrm{Bar}=5 \mu \mathrm{m}$; Hematoxylin counterstain. 
casionally, within the karyomegalic neoplastic cells in 2 livers and 9 spleens. The inclusions were observed in both the small and large bizarre lymphocytes (Fig. 2A and 2B).

The distribution pattern of the neoplastic lymphocytic accumulation within the affected spleens and livers was histopathologically divided as follows.

1) Multifocal lymphocytic accumulation: This pattern was noticed in $32 \%(12 / 37)$ of the total numbers of spleens with neoplastic lymphocytic accumulation and 69\% (9/13) of the total number of livers. In this pattern, the lymphocytic accumulation appeared as multiple, circumscribed and variably sized nodules within the parenchyma. The eosinophilic intranuclear inclusion bodies were noticed in the neoplastic cells of 3 out of 9 of spleens and in 2 out of 2 livers.

2) Coalesced lymphocytic accumulation: This pattern was noticed in $43 \%(16 / 37)$ of the total number of spleens with neoplastic lymphocytic accumulation and 23\% (3/13) of the total number of livers. In this pattern, the infected spleens showed uncircumscribed accumulations of neoplastic lymphocytes within the splenic parenchyma and these lesions were divided by thin indigenous splenic pulps, whereas the infected livers showed a severe invasion of uncircumscribed and variably sized foci in the hepatic sinusoids and hepatic parenchyma. Additionally, different degrees of hepatocyte necrosis were observed. The eosinophilic intranuclear inclusion bodies were observed in the neoplastic cells of 4 out of 9 infected spleens, while in the liver, no eosinophilic intranuclear inclusion bodies were noted.

3) Diffuse lymphocytic infiltration: This pattern represented $24 \%(9 / 37)$ of the total numbers of spleens with neoplastic lymphocyte infiltrations. In this pattern, the normal structure of the spleen was lost, and most of the splenic tissue was replaced by pleomorphic neoplastic lymphocytes. The livers did not show this pattern. The eosinophilic intranuclear inclusion bodies were observed in the neoplastic cells in 2 out of 9 infected spleens.

Immunohistochemistry: The CAV antigen was detected in the neoplastic cells of $24 \%(9 / 37)$ of the spleens with the neoplastic lymphocyte infiltrations and 15\% (2/13) of livers. The eosinophilic intranuclear inclusion bodies in the small and the large bizarre lymphocytes were positive for this antigen (Fig. 3A and 3B). RAV2 antigen was not detected in any of the tested organs.

\section{DISCUSSION}

Although the presence of coinfection of MDV and CAV was proved in several experimental studies [10, 11, 16], herein, we evaluated the existence of CAV antigen and its inclusions within naturally occurring lymphomas in condemned organs.

In this study, we confirmed MD lymphoma in 37 spleens and 12 livers based on the cellular morphology and infiltrative nature of the neoplastic lymphocytes. These lymphomas were composed of pleomorphic neoplastic lymphocytes and lymphoblasts with nuclear pleomorphism; these findings were consistent with previous reports [2, 18]. However, CAV inclusions were observed in the MD lymphomas of
9 spleens and 2 livers. CAV inclusions were observed in 2 types of neoplastic lymphocytes, the large bizarre cells and the small sized lymphocytes. Furthermore, these results were confirmed using immunohistochemistry with CAV antisera. These results suggest the possibility of the existence of CAV antigen within the MDV lymphomas in $>4$-week-old chickens, and this finding supports the experimental results of Haridy et al. [11] and Imai et al. [12]. The MD lymphomas, according to their pathomorphology, were divided into multifocal, coalesced and diffuse lymphocytic accumulations. CAV inclusions were detected in both multifocal and the coalesced patterns in a higher ratio than in the diffuse pattern. Because the 3 patterns are considered as sequential infiltrations of the neoplastic lymphocytes, the increase in the infiltration pattern of the MD neoplastic lymphocytes appears to have a negative correlation with the existence of CAV within the MDV lymphoma.

The pathogenesis of the co-infection with both the viruses is still poorly understood. However, we speculate the relationship between CAV and MDV during dual infection as symbiotic. CAV-neutralizing antibodies were previously believed to clear the virus [14]; However, recent studies detected chicken anemia viral DNA in gonadal tissues and spleens despite the flock seroconversion $[4,5]$. This may play a role in CAV latency in the offspring after reactivation of the virus during the sexual maturity of chickens [14]. This mechanism may result in a subclinical form of CAV, which causes an immunosuppressive effect and impairment in the generation of cytotoxic T lymphocytes [14] and in turn, influences the development of other diseases [17]. Based on the above-mentioned hypothesis, we assume that the poor vaccine-induced protection against MD and the late breakdown of MD vaccination might be caused by the reactivation of CAV. Moreover, the existence of CAV antigen and inclusions is closely correlated with the fact that CAV needs dividing cells for its replication $[1,20]$. The neoplastic cells in MD lymphomas are considered as uncontrolled dividing cells, which are susceptible to infection with CAV. The neoplastic cells of MD lymphomas may act as a nest for the replication and multiplication of CAV.

In conclusion, to our knowledge, this is the first report regarding the persistence of $\mathrm{CAV}$ antigen and its inclusions in naturally occurring MD lymphomas in >3-week-old chickens, and these MD lymphomas may act as a nest for $\mathrm{CAV}$, prolonging its dissemination time and increasing the possibility of further infection in chickens.

\section{REFERENCES}

1. Adair, B. M. 2000. Immunopathogenesis of chicken anemia virus infection. Dev. Comp. Immunol. 24: 247-255. [Medline] [CrossRef]

2. Burgess, S. C. 2004. Marek's disease lymphomas. pp. 98-111. In: Marek's Disease, an Evolving Problem. (Davison, F. and Nair, V. eds.), Elsevier Academic Press, London.

3. Calnek, B. W. 1980. Marek's disease virus and lymphoma. pp. 103-143. In: Oncogenic Herpesviruses, 1st ed. (Rapp, F. ed.), CRC Press, Boca Raton.

4. Cardona, C., Lucio, B., O’Connell, P., Jagne, J. and Schat, K. A. 
2000. Humoral immune responses to chicken infectious anemia virus in three strains of chickens in a closed flock. Avian Dis. 44: 661-667. [Medline] [CrossRef]

5. Cardona, C. J., Oswald, W. B. and Schat, K. A. 2000. Distribution of chicken anaemia virus in the reproductive tissues of specific-pathogen-free chickens. J. Gen. Virol. 81: 2067-2075. [Medline] [CrossRef]

6. Davidson, I. 2008. Interactions between Marek's disease virus and other pathogens. In The 8th International Marek's Disease Symposium. Townsville.

7. Davidson, I., Raibshtein, I. and Al-Touri, A. 2013. Quantitation of Marek's disease and chicken anemia viruses in organs of experimentally infected chickens and commercial chickens by multiplex real-time PCR. Avian Dis. 57 Suppl: 532-538. [Medline] [CrossRef]

8. Fadly, M. A. 2008. Neoplastic Diseases. pp. 449-451. In: Diseases of Poultry, 12th ed. (Saif, Y. M., Fadly, A. M., Glisson, J. R., McDougald, L. R., Nolan, L. K. and Swayne, D. E. eds.), Blackwell Publishing Professional, Ames.

9. Fehler, F. and Winter, C. 2001. CAV infection in older chickens: an apathogenic infection? pp. 391-394 In: International Symposium on Infectious Bursal Disease and Chicken Infectious Anemia. Rauischolzhausen.

10. Haridy, M., Goryo, M., Sasaki, J. and Okada, K. 2009. Pathological and immunohistochemical study of chickens with coinfection of Marek's disease virus and chicken anaemia virus. Avian Pathol. 38: 469-483. [Medline] [CrossRef]

11. Haridy, M., Sasaki, J., Okada, K. and Goryo, M. 2012. Persistence of inclusions and antigens of chicken anemia virus in Marek's disease lymphoma. Res. Vet. Sci. 93: 1353-1360. [Medline] [CrossRef]

12. Imai, K., Mase, M., Tsukamoto, K., Hihara, H. and Yuasa, N. 1999. Persistent infection with chicken anaemia virus and some effects of highly virulent infectious bursal disease virus infection on its persistency. Res. Vet. Sci. 67: 233-238. [Medline] [CrossRef]

13. Kreager, K. 1997. Marek's disease: Clinical aspects and current field problems in layer chickens. pp. 23-26. In: Diagnosis and Control of Neoplastic Diseases of Poultry. (Fadly, A. M., Schat, K. A. and Spencer, J. L. eds.), American Association of Avian
Pathologists., Kennett Square.

14. Markowski-Grimsrud, C. J. and Schat, K. A. 2003. Infection with chicken anaemia virus impairs the generation of pathogenspecific cytotoxic T lymphocytes. Immunology 109: 283-294. [Medline] [CrossRef]

15. McConnell, C. D., Adair, B. M. and McNulty, M. S. 1993. Effects of chicken anemia virus on cell-mediated immune function in chickens exposed to the virus by a natural route. Avian Dis. 37: 366-374. [Medline] [CrossRef]

16. Miles, A. M., Reddy, S. M. and Morgan, R. W. 2001. Coinfection of specific-pathogen-free chickens with Marek's disease virus (MDV) and chicken infectious anemia virus: effect of MDV pathotype. Avian Dis. 45: 9-18. [Medline] [CrossRef]

17. Miller, M. M. and Schat, K. A. 2004. Chicken infectious anemia virus: an example of the ultimate host-parasite relationship. Avian Dis. 48: 734-745. [Medline] [CrossRef]

18. Payne, L. N. and Venugopal, K. 2000. Neoplastic diseases: Marek's disease, avian leukosis and reticuloendotheliosis. Rev. - Off. Int. Epizoot. 19: 544-564. [Medline]

19. Schat, K. A. and Nair, V. 2013. Neoplastic Diseases. pp. 513673. In: Diseases of Poultry, 13th ed. (Swayne, D. E., Glisson, J. R., McDougald, L. R., Nolan, L. K., Suarez, D. L. and Nair, V. L. eds.), John Wiley \& Sons, Inc., Ames.

20. Schat, K. A. and van Santen, V. L. 2013. Chicken Infectious Anemia. pp. 248-264. In: Diseases of Poultry, 13th ed. (Swayne, D. E., Glisson, J. R., McDougald, L. R., Nolan, L. K., Suarez, D. L. and Nair, V. L. eds.), John Wiley \& Sons, Inc., Ames.

21. Smyth, J. A., Moffett, D. A., Connor, T. J. and McNulty, M. S. 2006. Chicken anaemia virus inoculated by the oral route causes lymphocyte depletion in the thymus in 3-week-old and 6-weekold chickens. Avian Pathol. 35: 254-259. [Medline] [CrossRef]

22. Sommer, F. and Cardona, C. 2003. Chicken anemia virus in broilers: dynamics of the infection in two commercial broiler flocks. Avian Dis. 47: 1466-1473. [Medline] [CrossRef]

23. Witter, R. L. 2001. Protective efficacy of Marek's disease vaccines. Curr. Top. Microbiol. Immunol. 255: 57-90. [Medline]

24. Yuasa, N., Taniguchi, T. and Yoshida, I. 1979. Isolation and some characteristics of an agent inducing anemia in chicks. Avian Dis. 23: 366-385. [CrossRef] 